\title{
Sporadic aurora from Spain
}

\author{
J. M. Vaquero ${ }^{1}$, R. M. Trigo ${ }^{2,3}$, and M. C. Gallego ${ }^{4}$ \\ ${ }^{1}$ Departamento de Física Aplicada, Escuela Politécnica, Universidad de Extremadura, Cáceres, Spain \\ ${ }^{2}$ University of Lisbon, CGUL, IDL, Lisbon, Portugal \\ ${ }^{3}$ Departamento de Enghenharias e Ciências Naturais, Universidade Lusófona, Lisbon, Portugal \\ ${ }^{4}$ Departamento de Física, Facultad de Ciencias, Universidad de Extremadura, Badajoz, Spain
}

(Received October 16, 2007; Revised November 15, 2007; Accepted November 16, 2007; Online published November 22, 2007)

\begin{abstract}
Some authors have shown recently that "sporadic auroras" have occurred at relatively low latitude when the geomagnetic activity is quiet or moderate. Here we present and analyze a case of "sporadic aurora" observed from Spain on the 29 December 1845 and compare its low geomagnetic activity with other three aurorae also observed in Spain in mid-19th century.
\end{abstract}

Key words: Sporadic aurora, low geomagnetic activity.

\section{Introduction}

Low-latitude auroral displays are relatively rare and usually associated with great solar storms such as the 1921 (Silverman and Cliver, 2001) and 1989 (Allen et al., 1989; Livesey, 1990) episodes. Nevertheless, low-latitude aurorae have been observed also during periods of weak-tomoderate geomagnetic activity, and this phenomenon has been described and catalogued for the North America (Silverman, 2003) and East Asia (Willis et al., 2007) regions. Silverman (2003) showed and analyzed US auroral data from 1880 to 1940 to demonstrate the reality of an auroral phenomenon with two important characteristics: (a) it occurred under conditions of quiet or moderate magnetic activity and (b) it occurred at low-latitudes where auroras normally do not occur except during great magnetic storms. Silverman (2003) used the term "Sporadic aurora" previously adopted in the literature dedicated to this rare type of aurora (Abbe, 1895; Botley, 1963). According to Willis et al. (2007) the word "sporadic aurorae" may be used to describe localised auroral displays that occur at low geomagnetic latitudes (approximately, $-45^{\circ} \leq$ geomagnetic latitude $\leq+45^{\circ}$ ) during intervals of weak-to-moderate geomagnetic activity $(a a \leq 50)$. Far eastern Asian countries have kept good records of low latitude auroras observed in the last millennium, namely over Japan (Nakazawa et al., 2004), Korea (Zhang, 1985) and China (Yau et al., 1995). Recently, Willis et al. (2007) have compiled 42 Chinese and Japanese auroral observations from 1840 to 1911 . They have demonstrated that at least 29 out of the 42 Chinese and Japanese auroral observations (i.e. 69\%) occurred at times of weak-to-moderate geomagnetic activity ( $a a$ or $A k$ index $\leq 50)$.

Willis et al. (2007) have suggested the possible connection of sporadic aurorae with solar activity but further re-

Copy right(c) The Society of Geomagnetism and Earth, Planetary and Space Sciences (SGEPSS); The Seismological Society of Japan; The Volcanological Society of Japan; The Geodetic Society of Japan; The Japanese Society for Planetary Sciences; TERRAPUB. search is required to explain how they can be observed at low latitudes and at times of weak or moderate geomagnetic activity levels.

The aim of this short contribution is to describe and contextualize a highly probable low-latitude sporadic aurora observed from Spain, within a period characterised by weak magnetic activity. It should be stressed that, up to now there has been no identification of sporadic auroral events over Europe. In fact, the aggregated number of similar lowlatitude auroras that have occurred under weak-to-moderate magnetic activity is fairly small (Abbe, 1895; Botley, 1963; Silverman, 2003; Willis et al., 2007).

\section{Data}

Rico Sinobas (1855) compiled a catalogue of auroras observed in the Iberian Peninsula in the period 1701-1848 including records of 40 different auroras. The most important data in that catalogue were summarised in Vaquero et al. (2003). In particular, Vaquero et al. (2003) indicate that (1) the auroral data recorded by Rico Sinobas is reliable based on the available collected information regarding colour and azimuth and (2) there was a notable gap in the occurrence of observed auroras during the Dalton minimum (1800-1820 approximately).

Direct comparison between the dates of observed auroras and the geomagnetic activity can only be performed for those events that have occurred after 1844, when the magnetic index $A k$ become available (Nevanlinna and Kataja, 1993; Nevanlinna, 2004). The other important geomagnetic indicator usually employed in these studies is the $a a$ index that is only available from 1868 until the present (Mayaud, $1980)$. In any case, prior to 1840 , there are no appropriate quantitative measurements of geomagnetic activity (Willis et al., 2007). Therefore, the common period for the Rico Sinobas catalogue and reliable values of the $A k$ index is fairly small spanning between 1844 and 1848. We intend to evaluate the geomagnetic activity prior, during and after the aurora display relative to four distinct auroral episodes 
Table 1. Auroral observations from Spain during 1844-1848.

\begin{tabular}{cccc}
\hline Date & Location & Description & Known magnetic storms? \\
\hline 29 Dec 1845 & Valencia $(39.5 \mathrm{~N} \mathrm{0.4W)}$ & Reddish brightness in the horizon & NO \\
\hline 24 Oct 1847 & San Fernando $(36.5 \mathrm{~N} \mathrm{6.2W)}$ & $\begin{array}{c}\text { Aurora with maxima intensity } \\
\text { at NNW direction }\end{array}$ & $\begin{array}{c}\text { YES. Nevanlinna (2006) reports mag. } \\
\text { storms in Helsinki on the 23 and 24 }\end{array}$ \\
\hline 18 Oct 1848 & Valladolid (41.7N 4.7W) & Reddish brightness (later white) & NO \\
& & at NNW direction & YES. Nevanlinna (2006) reports mag. \\
& Coruña (43.4N 8.4W) & Auroral bow from NNW to NNE. & storms in Helsinki on the 17 and 18 \\
& Cartagena (37.6N 1.0W) & Colours white, green and red & \\
\hline
\end{tabular}

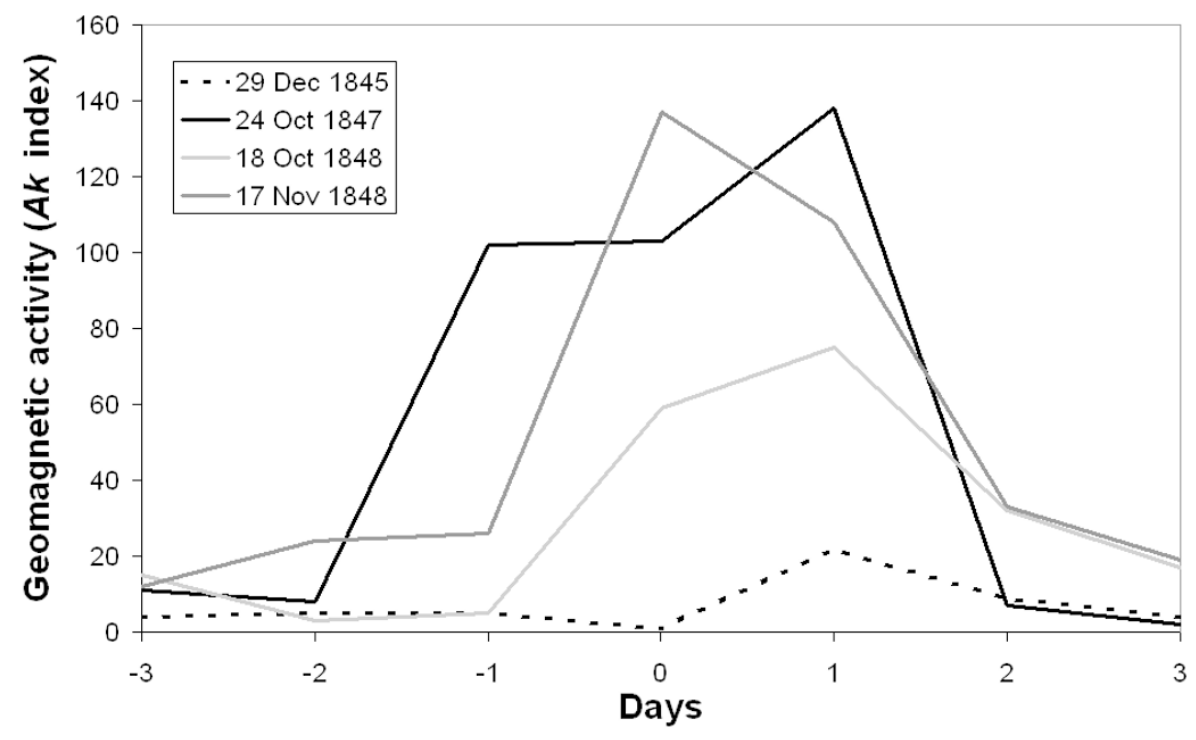

Fig. 1. Geomagnetic activity evolution (daily $A k$ index) for a 7-day interval centred on the day of the auroral observations recorded in the Rico Sinobas catalogue during the years 1844-1848.

described in Rico Sinobas catalogue for that common period (Table 1). We have also included information on the simultaneous detection of great magnetic storms in northern Europe (Nevanlinna, 2006). According to this list of storms, the auroras observed on the 24 Oct 1847 and 17 Nov 1848 correspond to major magnetic storms in northern Europe, with the $A k$ index values higher than 100 . On the contrary the events observed on 29 Dec 1845 and 18 Oct 1848 were not associated with a major storm.

\section{Results}

Figure 1 presents the geomagnetic activity evolution as measured by the daily $A k$ index for a period of seven days centred on the day when the aurora was observed in Spain during the considered period 1844-1848 (4 cases).

Concerning the three auroras that have occurred in 1847 and 1848, the geomagnetic activity has reached high levels $(A k>50)$ with the October 1847 and November 1848 episodes reaching considerably higher values $(A k>120)$. The aurora observed in October 1848 reveals a moderate-tohigh $A k$ index value (above 50 but below 80). This corroborates the fact that it was also not detected by Nevanlinna (2006) as a major storm in Helsinki (where the minimum threshold imposed by the author was $a a=100$ ).
However, the aurora that was observed on the 29 Dec 1845 occurred with very low values of the geomagnetic activity, i.e. the $A k$ index remained below 25 throughout the entire episode. Despite the fact that the geomagnetic activity was very low during the event of 1845 , one day after the event we can observe a somewhat higher value index $A k$ $(4,5,5,1,22,9,4)$. We should point out that Shiokawa et al. (2005) showed that some low-latitude auroras observed in Japan during the period 1999-2004 have occurred during the initial phase of magnetic storms. We believe that the low-latitude and low magnetic-activity event described here may correspond also to an early weak magnetic storm stage.

\section{Conclusion}

We are highly confident that the aurora observed from Spain on 29 Dec 1845 can be classified as a "sporadic aurora" because it was observed at relative low-latitude (Table 1) and during a weak period of geomagnetic activity (Fig. 1). To the best of our knowledge no work has been published describing sporadic auroral episode in Europe. In fact, apart from one observation (Eddie, 1894) from South Africa, all published episodes of sporadic aurorae have took place in the United States (Silverman, 2003) and East Asia (Willis et al., 2007). 
Unfortunately the lack of other available aurorae catalogs and the lack of systematic observations of auroras from Spain makes it impossible to increase the temporal coverage of this study.

Acknowledgments. The authors are in debt with Heikki Nevanlinna who has provided the daily $A k$ index values. J. M. Vaquero acknowledges the Programme "José Castillejo" from Spanish Science Ministry.

\section{References}

Abbe, C., An aurora in South Carolina and Kentucky, Mon. Weather Rev., 23, 297-298, 1895

Allen, J., H. Sauer, L. Frank, and P. Reiff, Effects of the March 1989 solar activity, Eos, 70, 1479, 1486-1488, 1989.

Botley, C. M., Sporadic aurora, Planet. Space Sci., 11, 723-724, 1963.

Eddie, L. A., Note on an auroral display, J. Brit. Astron. Assn., 4, 381, 1894.

Livesey, R. J., Aurora notes January-March 1989, The Marine Observer, 60, 40-45, 1990.

Mayaud, P. N., Derivation, Meaning, and Use of Geomagnetic Indices, Geophysical Monograph 22, American Geophysical Union, Washington, D.C., 1980.

Nakazawa, Y., T. Okada, and K. Shiokawa, Understanding the "SEKKI" phenomena in Japanese historical literatures based on the modern science of low-latitude aurora, Earth Planets Space, 56, e41-e44, 2004.

Nevanlinna, H., Results of the Helsinki magnetic observatory 1844-1912, Annales Geophysicae, 22, 1691-1704, 2004.
Nevanlinna, H., A study on the great geomagnetic storm of 1859: comparisons with other storms in the 19th century, Advances in Space Research, 38, 180-187, 2006.

Nevanlinna, H. and E. Kataja, An extension of the geomagnetic activity index series a for two solar cycles (1844-1868), Geophys. Res. Lett., 20, 2703-2706, 1993.

Shiokawa, K., T. Ogawa, and Y. Kamide, Low-latitude auroras observed in Japan: 1999-2004, J. Geophys. Res., 110, A05202, doi:10. 1029/2004JA010706, 2005.

Silverman, S. M., Sporadic auroras, J. Geophys. Res., 108(A4), 8011, doi: 10.1029/2002JA009335, 2003.

Silverman, S. M. and E. W. Cliver, Low-latitude auroras: the magnetic storm of 14-15 May 1921, J. Atmos. Solar-Terr. Phys., 63, 523-535, 2001.

Vaquero, J. M., M. C. Gallego, and J. A. GarcIa, Auroras in the Iberian Peninsula (1700-1855) from Rico Sinobas' Catalogue, Journal of Atmospheric and Solar-Terrestrial Physics, 65, 677-682, 2003.

Willis, D. M., F. R. Stephenson, and H. Fang, Sporadic aurorae observed in East Asia, Annales Geophysicae, 25, 417-436, 2007.

Yau, K. K. C., F. R. Stephenson, and D. M. Willis, A Catalogue of Auroral Observations from China, Korea and Japan (193 BC-AD 1770), Rutherford Appleton Laboratory Technical Report RAL-TR-95-073, December 1995 .

Zhang, Z. W., Korean Auroral Records of the Period 1507-1747AD and the SAR Arcs, British Astronomical Assoc. Journal, 95, 205-210, 1985.

J. M. Vaquero (e-mail: jvaquero@unex.es), R. M. Trigo, and M. C. Gallego 